\title{
A new dominance intensity method to deal with ordinal information about a DM's preferences within MAVT
}

\author{
E.A. Aguayo ${ }^{\text {a,* }}$, A. Mateos $^{\mathrm{a}}$, A. Jiménez-Martín ${ }^{\mathrm{a}}$ \\ ${ }^{a}$ Departamento de Inteligencia Artificial, Universidad Politécnica de Madrid,Campus de \\ Montegancedo S/N, Boadilla del Monte, 28660 Madrid, Spain
}

\begin{abstract}
Dominance measuring methods are an approach to deal with complex decisionmaking problems with imprecise information. These methods are based on the computation of pairwise dominance values and exploit the information in the dominance matrix in different ways to derive measures of dominance intensity and rank the alternatives under consideration. In this paper we propose a new dominance measuring method to deal with ordinal information about decisionmaker preferences in both weights and component utilities. It takes advantage of the centroid of the polytope delimited by ordinal information and builds triangular fuzzy numbers whose distances to the crisp value 0 constitute the basis for the definition of a dominance intensity measure. Monte Carlo simulation techniques have been used to compare the performance of this method with other existing approaches.
\end{abstract}

\section{Introduction}

The additive model is widely used within multi-attribute value theory (MAVT) to rank alternatives in complex decision-making problems and it is considered a valid approach in many practical situations for the reasons described in $<20$; $29>$. The functional form of the additive model is

$$
v\left(A_{i}\right)=\sum_{j=1}^{n} w_{j} v_{j}\left(x_{i j}\right),
$$

where $x_{i j}$ is the performance over the attribute (or criterion) $X_{j}, j=1, \ldots, n$, for the alternative $A_{i}, i=1, \ldots, m$; and $v_{j}$ and $w_{j}$ are the value function and the weight for the attribute $X_{j}$, respectively. Note that $\sum_{j=1}^{n} w_{j}=1$ and $w_{j} \geq 0$.

\footnotetext{
This paper was supported by the Madrid Regional Government project S-0505/TIC/0230, the Spanish Ministry of Education and Science project TIN2008-06796-C04-02 and the Spanish Ministry of Science and Innovation project MTM2011-28983-C03-03.

*Corresponding author. Tel.: +34 91336 6596; fax: +34 913524819.

Email addresses: ea.aguayo@upm.es (E.A. Aguayo), amateos@fi.upm.es (A. Mateos), antonio.jimenez@upm.es (A. Jiménez-Martín)
} 
The information available in most real complex decision-making problems is not precise. Inputs are often described within prescribed bounds or just satisfying certain relations. Different authors refer to this situation as decisionmaking with imprecise information, incomplete information or partial information $<21 ; 22>$.

Several reasons are given in the literature that justify why a decision-maker (DM) may wish to provide imprecise information $<27 ; 33>$. For example, performances that reflect social or environmental impacts may be intangible or non-monetary, and performances may be taken from statistics or measurements, which are not absolutely precise. Alternatively, DM might prefer not to reveal his/her preferences in public or not feel confident about giving precise information for parameters that change during the process. Besides, DMs could feel more comfortable providing a scale to represent the importance of the attributes, and might also have different more or less reliable sources of information. Moreover, the decision could be taken in a group decision-making situation, where a negotiation process usually outputs imprecise information $<6 ; 13 ; 17 ; 32\rangle$

Many papers on MAVT have dealt with imprecise information. Sarabando and Dias $<26>$ provided a brief overview of approaches proposed by different authors within the multi-attribute utility theory (MAUT) and MAVT framework to deal with imprecise information.

As attribute weights are usually the hardest parameters to elicit in multiattribute decision making (MADM) problems $<23>$, works in the literature have mainly centered on the case in which the information regarding weights is imprecise, which is often represented by ordinal information.

Surrogate weighting $(S W)$ methods can be used when the DM provides ordinal relations regarding attribute weights. These methods select a weight vector from a set of admissible weights to represent the set $<3 ; 30>$. The best $S W$ method is the rank-order centroid weights (ROC) method $<26>$ : $w_{j}=\frac{1 / j}{\sum_{k=1}^{n} 1 / k}, j=1, \ldots, n, n$ being he number of attributes.

The stochastic multicriteria acceptability analysis (SMAA) method was proposed for support in discrete group decision-making problems where the weight information is missing $<8>$. The $S M A A-2$ method $<10>$ extends the analysis to the sets of weight vectors for any rank from best to worst for each decision alternative and can be used to identify good compromise alternatives. SMAA$\mathrm{O}<9>$ is a variant of SMAA for problems in which criteria are measured on ordinal scales.

The TOPSIS method has been extended to uncertain linguistic environments $<35$; 36 $>$ or used for determining DM weights with interval numbers $<41>$.

Sage and White $<24>$ proposed the model of imprecisely specified multiattribute utility theory (ISMAUT), where preference information about both weights and utilities is assumed not to be precise. Malakooti $<11>$ suggested an efficient algorithm for ranking alternatives when there is imprecise information about preferences and alternative values. Ahn $<1>$ extended Malakooti's work.

Another possibility described in the literature for dealing with imprecision is 
based on the concepts of pairwise and absolute dominance. The use of absolute dominance values is exemplified by the modification of four classical decision rules to encompass an imprecise decision context concerning weights and component values/utilities $\langle 19 ; 25\rangle$, the maximax or optimist, the maximin or pessimist, the minimax regret and the central value rules.

A recent approach for dealing with imprecise information is to compute different measures of dominance to derive a ranking of alternatives $<2>$, known as dominance measuring methods (DMMs). DMMs are based on the computation of a dominance matrix including pairwise dominance values, which are exploited in different ways to derive measures of dominance to rank the alternatives under consideration.

In this paper we propose a new $D M M$ based on a dominance intensity measure to deal with ordinal information about the DM's preferences. Specifically, the DM will provide a ranking of attribute importance. Besides, the method takes into account a ranking of the alternatives in each attribute and also a ranking of the difference of values between consecutive alternatives.

As mentioned above, many methods accounting for ordinal information on weights and alternative values/utilities within MAVT/MAUT can be found in the literature. However, the ranking of the difference between the values of consecutive alternatives used to represent DM preferences is not so commonplace in the literature. Sarabando and Dias $<27>$ propose new decision rules within MAVT to deal with such rankings on the basis of an additive model, whereas Salo and Hamalainen $<25>$ transform them into linear constraints in the preference ratios in multiattribute evaluation PRIME method. In PRIME, preference elicitation and synthesis is based on 1) the conversion of possibly imprecise ratio judgments into an imprecisely specified preference model, 2) the use of dominance structures and decision rules in deriving decision recommendations, and 3 ) the sequencing of the elicitation process into a series of elicitation tasks.

Ordinal information has also been used in other disciplines apart from MAVT/ MAUT, for instance in fuzzy preference relations. Xu et al. $<39>$ propose the ordinal consistency index to measure the degree of ordinal consistency of a fuzzy preference relation, which is to count the unreasonable 3-cycles in a directed graph that represents the fuzzy preference relation. The method can be used for a strict and non-strict fuzzy preference relation. Xu et al. $<37 ; 40 ; 38>$ adapt the algorithms for incomplete reciprocal, inter-valued fuzzy and incomplete 2-tuple fuzzy linguistic preference relations, respectively.

The proposed dominance intensity measure takes advantage of the centroid of the polytope delimited by ordinal information, builds triangular fuzzy numbers on the basis of this centroid and incorporates a distance notion to derive dominance intensities to rank the alternatives under consideration.

We have also conducted a simulation study to analyze the performance of the proposed method regarding other dominance measuring methods proposed in the literature and Sarabando and Dias ranking method.

In Section 2, we review dominance measuring methods reported in the literature and the ranking method proposed by Sarabando and Dias. In Section 3, we propose the new dominance measuring method. In Section 4, we describe a 
technique to find all the endpoints from a polytope delimited by constraints representing ordinal information. We average the endpoints to derive the centroid of the polytope, which is used in the proposed dominance measuring method. In Section 5, a simulation study is carried out to compare the proposed method with the dominance measuring methods reviewed in Section 2 and the method proposed by Sarabando and Dias. Finally, some conclusions are discussed in Section 6 .

\section{Review of dominance measuring methods and Sarabando and Dias's method}

$D M M s$ are based on the computation of a dominance matrix, $D$, including pairwise dominance values:

$$
D=\left(\begin{array}{ccccc}
- & D_{12} & \cdots & D_{1(m-1)} & D_{1 m} \\
D_{21} & - & \cdots & D_{2(m-1)} & D_{2 m} \\
D_{31} & D_{32} & \cdots & D_{3(m-1)} & D_{3 m} \\
\vdots & \vdots & \vdots & \vdots & \vdots \\
D_{m 1} & D_{m 2} & \cdots & D_{m(m-1)} & -
\end{array}\right)
$$

where

$$
\begin{gathered}
D_{k l}=\min \left\{v\left(A_{k}\right)-v\left(A_{l}\right)=\sum_{j=1}^{n} w_{j} v_{k}\left(x_{k j}\right)-\sum_{j=1}^{n} w_{j} v_{l}\left(x_{l j}\right)\right\} \\
\text { s.t. } \\
\mathbf{v}_{k}=\left(v_{k 1}, \ldots, v_{k n}\right), \mathbf{v}_{l}=\left(v_{l 1}, \ldots, v_{l n}\right) \in V_{k l} \\
\mathbf{w}=\left(w_{1}, \ldots, w_{n}\right) \in W
\end{gathered}
$$

where $W$ and $V_{k l}$ define the feasible region for weights and values associated with the alternatives $A_{k}$ and $A_{l}$ over each attribute, respectively, which represent imprecise information.

Note that given two alternatives $A_{k}$ and $A_{l}$, alternative $A_{k}$ dominates $A_{l}$ if $D_{k l} \geq 0$, and there exists at least one $\mathbf{w}, \mathbf{v}_{k}$ and $\mathbf{v}_{l}$ such that the overall value of $A_{k}$ is strictly greater than that of $A_{l}$. This concept of dominance is called pairwise dominance.

The $D M M s$ exploit the information in $D$ in different ways to derive measures of dominance to rank the alternatives under consideration. For instance, Ahn and Park $<2>$ compute a dominating measure $\phi_{k}^{+}=\sum_{\substack{l=1 \\ l \neq k}}^{m} D_{k l}$ and a dominated measure $\phi_{k}^{-}=\sum_{\substack{l=1 \\ l \neq k}}^{m} D_{l k}$ for each alternative $A_{k}$, and then derive a net dominance as $\phi_{k}=\phi_{k}^{+}-\phi_{k}^{-}$. Ahn and Park proposed two ranking methods for these measures: ranking the alternatives according to either $\phi_{k}^{+}$or $\phi_{k}$ values (denoted as the $A P 1$ and $A P 2$ methods, respectively).

However, the results of simulation experiments when the DM weight preferences are represented by ordinal information suggest that surrogate weighting 
methods, specifically the $R O C$ method, are better than $A P 1$ and $A P 2$ at selecting the best alternative and ranking alternatives. The simulation study also showed $A P 1$ to be better than $A P 2$. The reason is that $A P 2$ uses duplicate information (row and column values).

Two DMMs were proposed in $<14 ; 15\rangle$. The first one, DME1, was based on the same idea as implemented by Ahn and Park. It also computes dominating and dominated measures but they are combined into a dominance intensity rather than a net dominance index, which is used as a measure of the strength of preference.

$D M E 1$ is implemented as follows:

1. Compute the dominating indices $D I_{k+}^{\text {row }}$ and $D I_{k-}^{\text {row }}$ for each alternative $A_{k}$ (by row):

$$
D I_{k+}^{\text {row }}=\sum_{l=1, l \neq k, D_{k l}>0}^{m} D_{k l} \text { and } D I_{k-}^{\text {row }}=\sum_{l=1, l \neq k, D_{k l}<0}^{m} D_{k l} .
$$

2. Compute the dominating intensity $D I_{k}^{\text {row }}$ for each alternative $A_{k}$ :

$$
D I_{k}^{\text {row }}=\frac{D I_{k+}^{\text {row }}}{D I_{k+}^{\text {row }}-D I_{k-}^{\text {row }}}
$$

3. Compute dominated indices $D I_{k+}^{c o l}$ and $D I_{k-}^{c o l}$ for each alternative $A_{k}$ (by column):

$$
D I_{k+}^{c o l}=\sum_{l=1, l \neq k, D_{l k}>0}^{m} D_{l k} \text { and } D I_{k-}^{c o l}=\sum_{l=1, l \neq k, D_{l k}<0}^{m} D_{l k} .
$$

4. Compute the dominated intensity $D I_{k}^{c o l}$ for each alternative $A_{k}$ :

$$
D I_{k}^{c o l}=\frac{D I_{k+}^{c o l}}{D I_{k+}^{c o l}-D I_{k-}^{c o l}}
$$

5. Calculate a global dominance intensity $(G D I)$ for each alternative $A_{k}$ :

$$
G D I_{k}=D I_{k}^{r o w}-D I_{k}^{c o l}, k=1, \ldots, m
$$

and rank the alternatives according to the $G D I_{k}$ values, where the alternative with the maximum $G D I_{k}$ is the best alternative.

$D M E 1$ improves $A P \mathscr{2}$ by reducing the duplicate information involved in the computations.

The second method, DME2, derives a global dominance intensity index to rank alternatives on the basis that

$$
D_{k l} \leq \mathbf{w}^{T}\left(\mathbf{v}_{k}-\mathbf{v}_{l}\right) \leq-D_{l k}, \forall \mathbf{w} \in W, \mathbf{v}_{k}, \mathbf{v}_{l} \in V_{k l}
$$

DME2 is implemented as follows: 
1. If $D_{k l} \geq 0$, then alternative $A_{k}$ dominates $A_{l}$, and the dominance intensity of $A_{k}$ over $A_{l}\left(D I_{k l}\right)$ is 1, i.e., $D I_{k l}=1$.

Else $\left(D_{k l}<0\right)$ :

- If $D_{l k} \geq 0$, then alternative $A_{l}$ dominates $A_{k}$, and $D I_{k l}=0$.

- Else $\left(D_{l k}<0\right)$, the dominance intensity of $A_{k}$ over $A_{l}$, is defined as

$$
D I_{k l}=\frac{-D_{l k}}{-D_{l k}-D_{k l}} .
$$

2. Calculate a global dominance intensity $(G D I)$ for each alternative $A_{k}$, i.e.,

$$
G D I_{k}=\sum_{l=1, l \neq k}^{m} D I_{k l},
$$

and rank the alternatives according to the $G D I_{k}$ values, where the alternative with the maximum $G D I_{k}$ is the best alternative.

Another simulation study $<16>$ was carried out to compare the DME1 and $D M E 2$ methods with modified decision rules (maximax, maximin and minimax regret and the central value rules) and $A P 1$ and AP2. Two measures of efficacy were considered, the proportion of all cases in which the method selects the same best alternative as in the TRUE ranking (hit ratio), where the TRUE ranking is determined beforehand, and how similar the overall alternative-ranking structures are in the TRUE and the method-driven rankings (rank-order correlation). The results show that DME2 outperforms the other methods. The drawback of the $D M E 1$ method is that when the dominance matrix $D$ contains all negative elements, that is, when all the alternatives are non-dominated, the algorithm is unable to rank the alternatives.

These methods in $<16>$ were adapted to account for imprecision concerning the inputs represented by value intervals, in alternative performances, component utilities and weights. The results of simulation studies $<16>$ showed that DME2 performs better than the AP1 method and the adaptation of classical decision rules and comes quite close to the $R O C$ method, which was identified as the best approach. Although SMAA-2 slightly outperforms DME2, DME2 could be used when incomplete information about weights is expressed not just as weight intervals but also as weights satisfying linear or non-linear constraints, weights represented by fuzzy numbers or weights fitting normal probability distributions.

The performance of DME1 and DME2 is compared in $<13>$ with other existing approaches ( $S W$ methods, modified decision rules and the AP1 and AP2 methods) when ordinal information represents imprecision concerning weights. As regards average hit ratios, DME2 and ROC outperform the other methods and, according to the paired-samples t-test, there is no significant difference between the two. However, $R O C$ can be only applied when ordinal relations regarding attribute weights are provided.

Other dominance measuring method was proposed in $\langle 7\rangle$ where imprecise weights are represented by trapezoidal fuzzy weights. Dominance values 


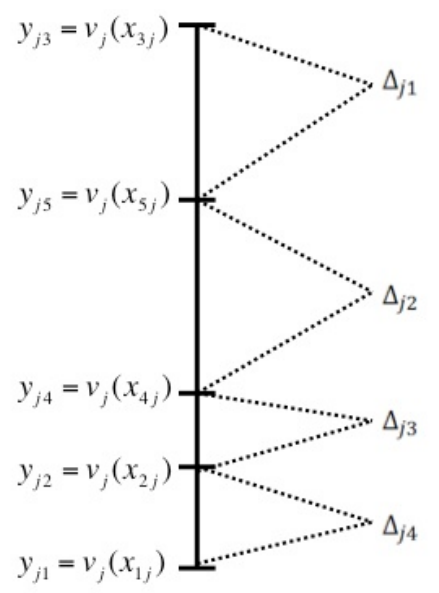

Figure 1: Ranking of alternatives and differences between consecutive alternatives for the attribute $X_{j}$.

are transformed into dominance intensity measures taking into account the distance between fuzzy numbers based on the generalization of the left and right fuzzy numbers defined by Tran and Duckstein $<31>$. An example concerning the selection of intervention strategies to restore an aquatic ecosystem contaminated by radionuclides illustrates the approach, and Monte Carlo simulation techniques are again used to analyze its performance for different imprecision levels.

As mentioned above, the ordinal information about the DM preferences considered in Sarabando and Dias $<27>$ is the same as in this paper, i.e., a ranking of the alternatives in each attribute and also of the difference between the values of consecutive alternatives. Therefore, Sarabando and Dias's method can be used to analyze the performance of the proposed method.

We denote by $V_{j}$ the set of constraints concerning component values in attribute $X_{j}$. For instance, $A_{3}$ could be the best of five alternatives for attribute $X_{j}$ for the DM, followed by $A_{5}, A_{4}, A_{2}$ and $A_{1}\left(v_{j}\left(x_{3 j}\right) \geq v_{j}\left(x_{5 j}\right) \geq v_{j}\left(x_{4 j}\right) \geq\right.$ $\left.v_{j}\left(x_{2 j}\right) \geq v_{j}\left(x_{1 j}\right)\right)$. Moreover, the ranking of differences between consecutive alternatives could be $\Delta_{j 2} \geq \Delta_{j 1} \geq \Delta_{j 4} \geq \Delta_{j 3}$, with $\Delta_{j 2}=v_{j}\left(x_{5 j}\right)-v_{j}\left(x_{4 j}\right)$, $\Delta_{j 1}=v_{j}\left(x_{3 j}\right)-v_{j}\left(x_{5 j}\right), \Delta_{j 4}=v_{j}\left(x_{2 j}\right)-v_{j}\left(x_{1 j}\right)$ and $\Delta_{j 3}=v_{j}\left(x_{4 j}\right)-v_{j}\left(x_{2 j}\right)$, as illustrated in Fig. 1.

Sarabando and Dias $<27>$ used the $R O C$ method to derive a weight vector. Besides, they propose an adaptation of the $R O C$ method, $\triangle R O C$, to compute a vector of values for each attribute that can approximately represent all the vectors' values compatible with the available ordinal information: 
1. Determine a rank order centroid for $m-1$ variables:

$$
\Delta_{j k}=\frac{1}{m-1} \sum_{l=\operatorname{rank}\left(\Delta_{j k}\right)}^{m-1} \frac{1}{l}, k=1, \ldots, m-1,
$$

where $\operatorname{rank}\left(\Delta_{j k}\right)$ is 1 when $\Delta_{j k}$ is the best, 2 when it is the second best, and so on.

2. The approximate values for the levels in attribute $X_{j}$, are:

$$
\begin{gathered}
y_{j t}=0, \text { if } \operatorname{rank}\left(y_{j t}\right)=m, \\
y_{j i}=\sum_{k=\operatorname{rank}\left(y_{j i}\right)}^{m-1} \Delta_{j k}, \text { if } i=1, \ldots, m, \text { and } \operatorname{rank}\left(y_{j i}\right) \neq m,
\end{gathered}
$$

where $\operatorname{rank}\left(y_{j i}\right)$ is 1 when $y_{j i}$ is the best, 2 when it is the second best, and so on.

For the example in Figure 1 with 5 alternatives, we have $\Delta_{j 2}=25 / 48, \Delta_{j 1}=$ $13 / 48, \Delta_{j 4}=7 / 48$ and $\Delta_{j 3}=1 / 16$. Then, $y_{j 1}=0, y_{j 2}=\Delta_{j 4}=7 / 48$, $y_{j 3}=\Delta_{j 1}+\Delta_{j 2}+\Delta_{j 3}+\Delta_{j 4}=1, y_{j 4}=\Delta_{j 3}+\Delta_{j 4}=10 / 48$ and $y_{j 5}=$ $\Delta_{j 2}+\Delta_{j 3}+\Delta_{j 4}=35 / 48$.

Finally, the additive model, see Eq. (1), is used to evaluate and rank the alternatives under consideration.

\section{A new dominance intensity method based on triangular fuzzy num- bers and a distance notion}

We consider that a DM's preferences are represented by ordinal information, for both weights and component values. Consequently, the DM provides a ranking of attribute importance. Without loss of generality we assume that attribute weights are indexed in descending order $\mathbf{w}=\left(w_{1}, w_{2}, \ldots, w_{n}\right) \in W: w_{1} \geq w_{2} \geq$ $\ldots \geq w_{n} \geq 0, \sum_{j=1}^{n} w_{j}=1$.

In this specific case, the optimization problem for deriving pairwise dominance values is non-linear, see Eq. (2), since it incorporates the product of pairs of variables (attribute weights and component values) in the objective function. We can simplify the problem by applying the $R O C$ method on the basis of the available ordinal information about weights. This has been demonstrated to derive a good representation of the set $W$, as cited in Section 2. The $R O C$ method is generalized to cases that include weak orders or partial orders in $\langle 28\rangle$. We denote by $\left(w_{1}^{c}, \ldots, w_{n}^{c}\right)$ the weight vector resulting from the $R O C$ method, which is the centroid of $W$.

The optimization problem is now linear since only the component values are under consideration. Thus, this problem could be solved using the simplex method, the dominance matrix $D$ and the dominance measuring methods ( $A P 1$, AP2, DME1 and DME2) applied to rank the considered alternatives. 
In this paper, we propose computing the following rather than pairwise dominance values $\left(D_{k l}\right)$ :

$$
v_{k l}=\sum_{j=1}^{n} w_{j}^{c} v_{k j}^{c}-\sum_{j=1}^{n} w_{j}^{c} v_{l j}^{c}
$$

where $\left(w_{1}^{c}, \ldots, w_{n}^{c}\right)$ is the centroid or center of gravity of the polytope representing the weight space and $\left(v_{k 1}^{c}, v_{l 1}^{c}\right), \ldots,\left(v_{k n}^{c}, v_{l n}^{c}\right)$ are the centroids or centers of gravity of the polytopes in the $n$ attributes delimited by the constraints accounting for alternatives $A_{k}$ and $A_{l}$. Note that the centroid is considered as the most representative point that verifies the constraints that delimit the polytope. Moreover, $D_{k l} \leq v_{k l} \leq-D_{l k}$.

The centroid of the polytope associated with constraints on component values in the attribute $X_{j}$ for the alternatives $A_{k}$ and $A_{l}$ is:

$$
\mathbf{v}_{j}^{c}=\left(v_{k j}^{c}, v_{l j}^{c}\right)=\frac{\int_{[0,1]^{2}} V_{j}^{k l} d v}{\int_{[0,1]^{2}} d v},
$$

where $V_{j}^{k l}$ is the set of constraints concerning component values in the attribute $X_{j}$ for alternatives $A_{k}$ and $A_{l}$. Note that $V_{j}^{k l} \subset V_{j}$, which includes the constraints concerning component values in the attribute $X_{j}$ for all the alternatives.

Some techniques have been proposed to find the center of gravity of a polytope, see, e.g., Lahdelma et al. $<8>$; Lahdelma and Salminen $<10>$; Mármol et al. $<12>$. In Section 4 we propose a method to derive the endpoints of a polytope delimited by constraints representing the ordinal information on component utilities. The centroid can then be computed by averaging these endpoints.

As it would be very simplistic to represent a constraint set as just a point, we have built a normalized triangular fuzzy number as follows. We assign possibility 1 to the value $v_{k l}$ and, as $D_{k l} \leq v_{k l} \leq-D_{l k}$, the possibility linearly decreases to $D_{k l}$ and $-D_{l k}$. However, as $v_{k l}$ is computed from centroids, a better option is to consider the following symmetric triangular fuzzy number (see Fig. 2):

$$
\widetilde{I}_{k l}=\left(I_{k l}^{L}, v_{k l}, I_{k l}^{U}\right),
$$

where $I_{k l}^{L}=v_{k l}-m_{k l}$ and $I_{k l}^{U}=v_{k l}+m_{k l}$, and

$$
m_{k l}=\min \left\{\left(-D_{l k}-v_{k l}\right),\left(v_{k l}-D_{k l}\right)\right\}
$$

with membership function (see Fig 2.)

$$
\mu_{\widetilde{I}_{k l}}(x)=\left\{\begin{array}{ll}
\frac{x-I_{k l}^{L}}{v_{k l}-I_{k l}^{L}}, & \text { if } I_{k l}^{L} \leq x \leq v_{k l} \\
1, & \text { if } x=v_{k l} \\
\frac{x-I_{k l}^{U}}{v_{k l}-I_{k l}^{U}}, & \text { if } v_{k l} \leq x \leq I_{k l}^{U} \\
0, & \text { otherwise }
\end{array} .\right.
$$



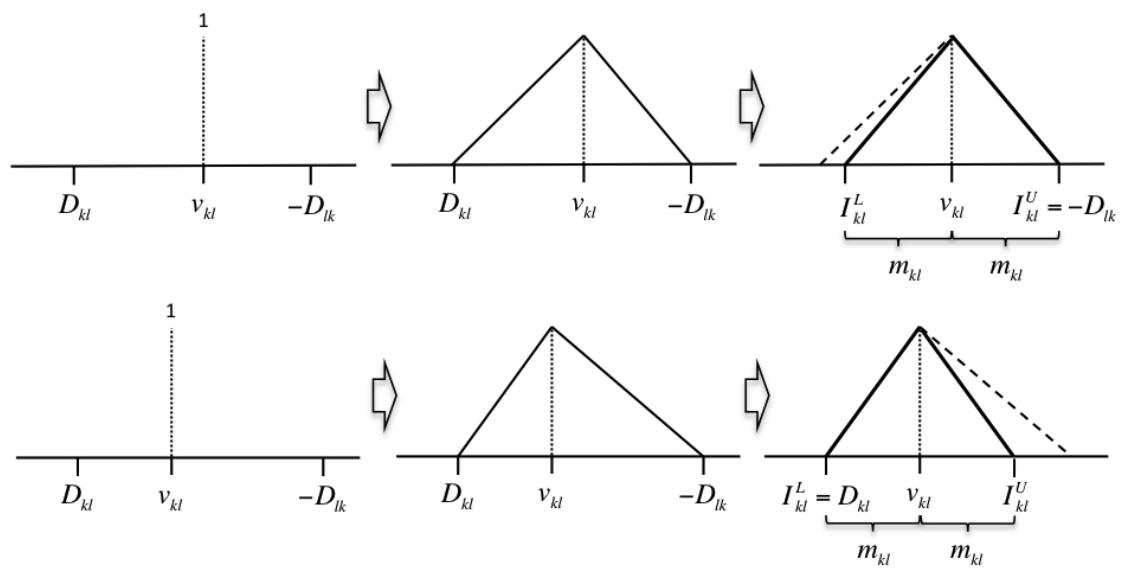

Figure 2: Building $\widetilde{I}_{k l}$.

Note that alternative $A_{k}$ is better than $A_{l}$ in the positive portion of the interval $I_{k l}$. However, alternative $A_{l}$ is better than $A_{k}$ in the negative portion.

Then, normalized triangular fuzzy numbers $\widetilde{I}_{k l}$ could be used in conjunction with a distance notion proposed in Tran and Duckstein $<31>$, to define a dominance intensity measure as follows: If we consider the location of the triangular fuzzy number $\widetilde{I}_{k l}$ regarding the crisp value 0 , then we have two possibilities (see Fig. 3): if $v_{k l}<0$, then the dominance intensity of alternative $A_{k}$ over $A_{l}$ can be computed as minus the distance of the fuzzy number $\widetilde{I}_{k l}$ to the crisp value 0 . Otherwise $\left(v_{k l} \geq 0\right)$, the dominance intensity is the distance of the fuzzy number $\widetilde{I}_{k l}$ to the crisp value 0 .

Note that in both cases we are already taking into account the possibility of $\widetilde{I}_{k l}$ being located completely on the right and on the left of zero, respectively, see cases $b$ ) and $d$ ) in Fig. 3. In the case $d$ ) alternative $A_{k}$ dominates $A_{l}$, whereas in the $b) A_{l}$ dominates $A_{k}$. This constitutes a difference with respect to the DME2 method, in which the dominance intensity of $A_{k}$ over $A_{l}$ is $1\left(D I_{k l}=1\right)$ when $D_{k l} \geq 0$ (alternative $A_{k}$ dominates $A_{l}$ ), whereas $D I_{k l}=0$ when $D_{l k} \geq 0$ (alternative $A_{l}$ dominates $A_{k}$ ). Therefore, it does not consider the strength of dominance, i.e., there is no difference between $D_{k l}=0.1$ or $D_{k l}=1.5$, where $D I_{k l}=1$ in both cases. However, we use the distance of $\widetilde{I}_{k l}$ to zero as the dominance intensity.

Finally, a dominance intensity measure for each alternative $A_{k}, D I M_{k}$, is derived as the sum of the dominance intensities of alternative $A_{k}$ regarding the other alternatives. This measure is used as a measure of the strength of preference in the sense that greater dominance intensity is better. 

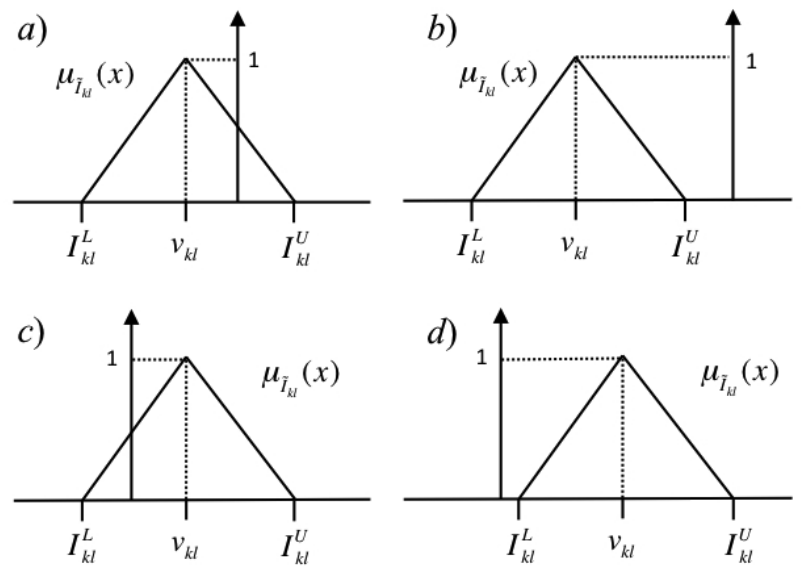

Figure 3: Locations of triangular fuzzy numbers.

Briefly, the method can be implemented as follows:

1. Compute values $v_{k l}$ by averaging the endpoints of the polytope delimited by constraints representing the ordinal information on component utilities using the method described in Section 4.

2. Build the triangular fuzzy numbers $\widetilde{I}_{k l}=\left(I_{k l}^{L}, v_{k l}, I_{k l}^{U}\right)$. To do this, first compute pairwise dominance values, $D_{k l}$, solving the optimization problem in Eq. (2). Note that the method proposed in Section 4 can again be used, since one of the endpoints is the optimal solution, which can be identified by just evaluating the endpoints in the objective function.

3. Compute the dominance intensities as follows:

- If $v_{k l} \geq 0$, then $D I_{k l}=d\left(\widetilde{I}_{k l}, 0, f\right)$, where $d$ refers to Tran and Duckstein's distance $<31>$, and $f$ is a weight function for differentiating a risk-averse, risk-neutral or risk-prone DM, as explained later.

- Else $\left(v_{k l}<0\right), D I_{k l}=-d\left(\widetilde{I}_{k l}, 0, f\right)$.

4. Compute a dominance intensity measure for each alternative $A_{k}$,

$$
D I M_{k}=\sum_{l=1, l \neq k}^{m} D I_{k l} .
$$

5. Rank alternatives according to $D I M_{k}$ values, where the alternatives with the maximum and minimum $D I M_{k}$ are the best and worst, respectively.

The distance defined by Tran and Duckstein $<31>$ for the generalization of left and right fuzzy numbers $(G L R F N)<4>$ is used in $d\left(\widetilde{I}_{k l}, 0, f\right)$. A fuzzy set $\tilde{a}=\left(a_{1}, a_{2}, a_{3}, a_{4}\right)$ is called a generalization of the left and right fuzzy numbers 
$(G L R F N)$ when its membership function is defined as

$$
\mu_{\widetilde{a}}(x)= \begin{cases}L\left(\frac{a_{2}-x}{a_{2}-a_{1}}\right), & \text { if } a_{1} \leq x \leq a_{2} \\ 1, & \text { if } a_{2} \leq x \leq a_{3} \\ R\left(\frac{x-a_{3}}{a_{4}-a_{3}}\right), & \text { if } a_{3} \leq x \leq a_{4} \\ 0, & \text { otherwise, }\end{cases}
$$

where $L$ and $R$ are strictly decreasing functions defined in [0,1] and satisfying the conditions:

$$
L(x)=R(x)=1 \text { if } x \leq 0 \text { and } L(x)=R(x)=0 \text { if } x>0 .
$$

Triangular fuzzy numbers are special cases of $G L R F N$ with $L(x)=R(x)=$ $1-x$ and $a_{2}=a_{3}$. A GLRFN is denoted as $\tilde{a}=\left(a_{1}, a_{2}, a_{3}, a_{4}\right)_{L \tilde{a}-R \tilde{a}}$ and an $\alpha$-cut of $\tilde{a}$ is a crisp set that contains all the elements that have a membership value greater than or equal to $\alpha$ :

$$
\tilde{a}(\alpha)=\left(\tilde{a}_{L}(\alpha), \tilde{a}_{R}(\alpha)\right)=\left(a_{2}-\left(a_{2}-a_{1}\right) a_{3} L_{\tilde{a}}^{-1}(\alpha), a_{3}-\left(a_{4}-a_{3}\right) a_{3} R_{\tilde{a}}^{-1}(\alpha)\right) .
$$

Tran and Duckstein $<31>$ define the distance between two GLFRN fuzzy numbers $\tilde{a}$ and $\widetilde{b}$ as

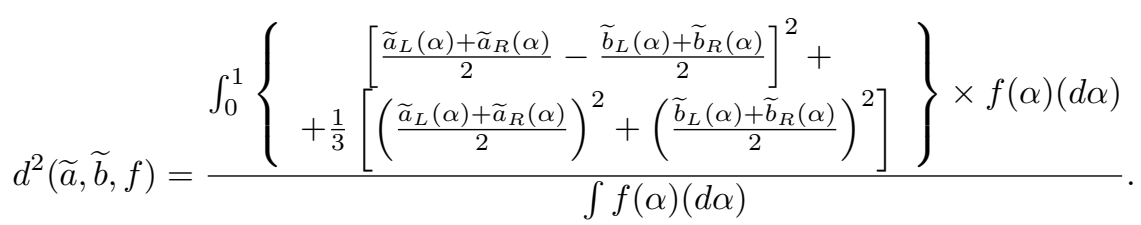

The function $f(\alpha)$ is positive continuous in $[0,1]$ and serves as a weight function. The distance is computed as the weighted sum of distances between two intervals across all $\alpha$-cuts from 0 to 1 . Moreover, it flexibilizes DM participation. For example, $f(\alpha)=\alpha$ looks to be reasonable when the DM is risk-neutral, whereas a risk-averse DM would put more weight on information at a higher $\alpha$ level by using functions such as $f(\alpha)=\alpha^{2}$ or a higher power of $\alpha$. A constant $(f(\alpha)=1)$, or even a decreasing function $f$, could be used for a risk-prone DM.

For the particular case of the distance from a triangular fuzzy number $\tilde{a}=$ $\left(a_{1}, a_{2}, a_{3}\right)$ to a constant (specifically 0$)$, we have:

1. If $f(\alpha)=\alpha$, then

$$
\begin{aligned}
d^{2}(\widetilde{a}, 0, f)= & a_{2}^{2}+\frac{1}{3} a_{2}\left(a_{3}+a_{1}\right)+\frac{1}{18}\left[\left(a_{3}-a_{2}\right)^{2}+\left(a_{2}-a_{1}\right)^{2}\right]- \\
& -\frac{1}{18}\left[\left(a_{2}-a_{1}\right)\left(a_{3}-a_{2}\right)\right] .
\end{aligned}
$$

2. If $f(\alpha)=1$, then

$$
\begin{aligned}
d^{2}(\widetilde{a}, 0, f)= & a_{2}^{2}+\frac{1}{2} a_{2}\left(a_{3}+a_{1}\right)+\frac{1}{9}\left[\left(a_{3}-a_{2}\right)^{2}+\left(a_{2}-a_{1}\right)^{2}\right]- \\
& -\frac{1}{9}\left[\left(a_{2}-a_{1}\right)\left(a_{3}-a_{2}\right)\right] .
\end{aligned}
$$


3. If $f(\alpha)=\alpha^{2}$, then

$$
\begin{aligned}
d^{2}(\widetilde{a}, 0, f)= & a_{2}^{2}+\frac{1}{4} a_{2}\left(a_{3}+a_{1}\right)+\frac{1}{144}\left[\left(a_{3}-a_{2}\right)^{2}+\left(a_{2}-a_{1}\right)^{2}\right]- \\
& -\frac{1}{96}\left[\left(a_{2}-a_{1}\right)\left(a_{3}-a_{2}\right)\right] .
\end{aligned}
$$

\section{Set of endpoints and centroid of a polytope delimited by con- straints representing ordinal information}

In this section we propose a method for deriving the set of endpoints of a polytope delimited by the following constraints:

1. A ranking of the variables under consideration $y_{1}, \ldots, y_{m} \in[0,1], y_{1} \geq$ $y_{2} \geq \ldots \geq y_{m}$.

2. A ranking of the differences between consecutive variables in the above ranking $\Delta_{j}=y_{j}-y_{j+1}, j=1, . ., m-1$.

First, we build the set of vertices for the polytope under consideration, denoted by $V$. The first vertex to be added to $V$ is $(0,0,0, \ldots, 0)$, since this vector satisfies all constraints. To build a new vertex we assign a value 1 to the position corresponding to the best-ranked variable in the above vertex. Next, we assign a value 1 to the position corresponding to the second ranked variable in the previous vertex, leading to a new vertex, and so on, until we reach the worst-ranked variable, which yields the vertex, $(1,1, \ldots, 1)$.

Note that when the variables are ranked in descending order $\left(y_{1} \geq y_{2} \geq\right.$ $\left.\ldots \geq y_{m}\right)$, then it is trivial to derive $V, V=\{(0,0,0,0, \ldots, 0),(1,0,0,0, \ldots, 0)$, $(1,1,0,0, \ldots, 0),(1,1,1,0, \ldots, 0), \ldots,(1,1,1,1 \ldots, 1)\}$.

Now, we consider the ranking of the differences between consecutive variables in the above variable ranking, $\Delta_{j}=y_{j}-y_{j+1}, j=1, . ., m-1$. We denote by $E P$ and $M$ the sets to which we add endpoints of the polytope and the differences between consecutive variables, respectively. Both sets are initially empty, and $E P$ will contain all the endpoints of the polytope when the procedure ends.

Then, we progressively add to $M$ the difference between consecutive variables according to the available ranking, i.e., first we add the best-ranked difference, then the second-ranked and so on. Each time a new difference is added to $M$, the vertices in $V$ associated with the best variable of each element in $M$ are averaged, and the resulting vector is added to $E P$. The procedure ends when all differences have been added to $M$, i.e., $M=\left\{\Delta_{1}, \Delta_{2}, \ldots, \Delta_{m-1}\right\}$. Finally, we add the endpoint $(0,0,0, \ldots, 0)$ to $E P$.

The algorithm for deriving the endpoints is as follows:

- Step 1. Build the set of vertices $V$ considering the ranking of the variables $y_{1}, \ldots, y_{m}$. Set $V=\emptyset$.

- Add $(0,0, \ldots, 0)$ to $V$.

- For $i=1, \ldots, m$ :

* Identify the $i$-th best-ranked variable in the ranking, $y_{j b e s t}$. 
* Assign value 1 to the element in the previous vertex added to $V$ corresponding to $y_{j b e s t}$.

* Add the new vertex to $V$.

Note that there will be $m+1$ elements in $V$ at the end of Step 1 .

- Step 2. We consider the ranking of the differences between consecutive variables $\Delta_{i}=y_{i}-y_{i+1}, j=1, \ldots, m-1$. Set $M=\emptyset$ and $E P=\emptyset$.

For $i=1, \ldots, m-1$ :

- Add the $i$-th best-ranked difference to $M$.

- Identify the vertices associated with the best variable of each element in $M$.

- Compute and add the average of the considered vertices to EP.

- Step 3. Add $(0,0, \ldots, 0)$ and $(1,1, \ldots, 1)$ to $E P$.

Finally, the EP set contains all the endpoints of the polytope, whose average yields to the centroid of the polytope.

Next, we illustrate the method with the example shown in Fig. 1, i.e., we consider a problem with five variables and the following rankings: $y_{3} \geq y_{5} \geq$ $y_{4} \geq y_{2} \geq y_{1}$ and $\Delta_{2} \geq \Delta_{1} \geq \Delta_{4} \geq \Delta_{3}$, with $\Delta_{1}=y_{3}-y_{5}, \Delta_{2}=y_{5}-y_{4}$, $\Delta_{3}=y_{4}-y_{2}$ and $\Delta_{4}=y_{2}-y_{1}$.

Then, the algorithm would work as follows:

- Step 1: From $y_{3} \geq y_{5} \geq y_{4} \geq y_{2} \geq y_{1}$, we have associated the vertices as follows: $V=\left\{(0,0,0,0,0), y_{3}:(0,0,1,0,0), y_{5}:(0,0,1,0,1)\right.$, $\left.y_{4}:(0,0,1,1,1), y_{2}:(0,1,1,1,1), y_{1}:(1,1,1,1,1)\right\}$.

- Step 2: $M=\emptyset$ and $E P=\emptyset$.

$i=1$ :

$-\Delta_{2}=y_{5}-y_{4}$ is the best-ranked difference, so $M=\left\{\Delta_{2}\right\}$.

$-y_{5}$ is the best variable corresponding to $\Delta_{2}$, then the vertex in $V$ corresponding to $y_{5},(0,0,1,0,1)$, is added to $E P$.

$i=2$ :

- $\Delta_{1}=y_{3}-y_{5}$ is the second-ranked difference, so $M=\left\{\Delta_{2}, \Delta_{1}\right\}$.

- $y_{5}$ and $y_{3}$ are the best variables corresponding to the differences in $M$, $\Delta_{2}$ and $\Delta_{1}$, respectively. We compute the average of the associated vertices, $y_{5}:(0,0,1,0,1)$ and $y_{3}:(0,0,1,0,0)$, which we add to $E P$. $E P=\{(0,0,1,0,1),(0,0,1,0,1 / 2)\}$.

$i=3$ :

- $\Delta_{4}=y_{2}-y_{1}$ is the third-ranked difference, so $M=\left\{\Delta_{2}, \Delta_{1}, \Delta_{4}\right\}$. 
$-y_{5}, y_{3}$ and $y_{2}$ are the best variables corresponding to the differences in $M$, respectively. We compute the average of vertices $y_{5}:(0,0,1,0,1)$, $y_{3}:(0,0,1,0,0)$ and $y_{2}:(0,1,1,1,1)$, which we add to $E P$.

$E P=\{(0,0,1,0,1),(0,0,1,0,1 / 2),(0,1 / 3,1,1 / 3,2 / 3)\}$.

$i=4$ :

- $\Delta_{3}=y_{4}-y_{2}$ is the worst-ranked difference, so $M=\left\{\Delta_{2}, \Delta_{1}, \Delta_{4}, \Delta_{3}\right\}$.

$-y_{5}, y_{3}, y_{2}$ and $y_{4}$ are now the best variables corresponding to the differences in $M$, respectively. We compute the average of vertices $y_{5}$ : $(0,0,1,0,1), y_{3}:(0,0,1,0,0), y_{2}:(0,1,1,1,1)$ and $y_{4}:(0,0,1,1,1)$, which we add to $E P$.

$E P=\{(0,0,1,0,1),(0,0,1,0,1 / 2),(0,1 / 3,1,1 / 3,2 / 3),(0,1 / 4,1,1 / 2$, $3 / 4)\}$.

- Step 3: $(0,0,0,0,0)$ and $(1,1,1,1,1)$ are added to EP.

Finally, $E P=\{(0,0,1,0,1),(0,0,1,0,1 / 2),(0,1 / 3,1,1 / 3,2 / 3),(0,1 / 4,1,1 / 2,3 / 4),(0$, $0,0,0,0),(1,1,1,1,1)\}$, and the centroid is derived by averaging the endpoints in $E P$, yielding $(1 / 6,19 / 72,5 / 6,11 / 36,47 / 72)$.

Note importantly that the method proposed in this section can also be used if we have the ranking of alternatives for each attribute under consideration, but the information about the differences between the values of consecutive alternatives is not available. This situation is less stressful on DMs and makes the method suitable for more real decision-making problems, in which the expert is often reluctant or may find it difficult to provide much information about his/her preferences. Notice also that the differences between the values of consecutive alternatives may be hard to quantify.

However, the method cannot be used if the available ordinal information is partial rather than complete, i.e., some alternatives are not included in the rankings available for some attributes.

\section{Performance analysis based on Monte Carlo simulation techniques}

In this section we analyze and compare the performance of the proposed method, $D I M$, with other dominance measuring methods (AP1, DME1 and DME2) and with the method proposed by Sarabando and Dias $<27>$, which represents the imprecision concerning the DM's preferences in the same way as in this paper.

We set out to carry out a simulation study for different scenarios accounting for different numbers of alternatives and attributes. In accordance with previous simulations performed in the literature, we identify six different levels for the alternatives $(m=3,5,7,10,15,20)$ and five different levels for the attributes $(n=3,5,7,10,15)$, yielding 30 design scenarios.

The process would be as follows for each scenario: 
1. Generate component values randomly from a uniform distribution in $(0,1)$, yielding an $m \times n$ matrix. This matrix has to be normalized making the smallest and largest values from each column zero and one, respectively. Note that dominated alternatives have to be removed in the simulation since they are not useful for analyzing the performance of the considered methods. From each row of the above matrix we derive the ranking of alternatives in each attribute and the ranking of the differences between consecutive alternatives.

2. Generate attribute weights randomly. First, we select $n-1$ independent random numbers from a uniform distribution on $(0,1)$, and rank these numbers. Suppose the ranked numbers are $1 \geq r_{n-1} \geq \ldots \geq r_{2} \geq r_{1}>0$. The differences between consecutive ranked numbers are then used as the target weights $w_{n}^{T}=1-r_{n-1}, w_{n-1}^{T}=r_{n-1}-r_{n-2}, \ldots, w_{1}^{T}=r_{1}$. The resulting weights will sum 1 and be uniformly distributed in the weight space $<5>$. They are used to derive the ranking of attribute weights. Note that these weights will be the TRUE weights. The TRUE ranking of alternatives is computed using the component value matrix from the previous step and the TRUE weights.

3. Compute a ranking of alternatives for each method according to their algorithms using just the ordinal information obtained from the component value matrix and weights.

4. Compare the rankings provided by each method with the TRUE ranking. We use two measures of efficacy, the hit ratio and the rank-order correlation $<2 ; 3>$. The hit ratio is the proportion of all cases in which the method selects the same best alternative as in the TRUE ranking. Rank-order correlation represents how similar the overall rank structures of alternatives are in the TRUE ranking and in the ranking derived from the method. It is calculated using Kendall's $\tau$ (Winkler and Hays $<34>$ ):

$$
\tau=1-\frac{2 \times(\text { number of pairwise preference violations })}{\text { total number of pair preferences }}=\frac{S}{m(m-1) / 2},
$$

where $S$ is the difference between the number of concordant (ordered equally) and discordant (ordered differently) pairs and $m$ is the total number of alternatives.

If there are tied (same value) observations then the denominator $m(m-$ 1)/2 has to be replaced by

$$
\sqrt{\left[m(m-1) / 2-\sum_{i=1}^{t} t_{i}\left(t_{i}-1\right) / 2\right]\left[m(m-1) / 2-\sum_{i=1}^{t} u_{i}\left(u_{i}-1\right) / 2\right]},
$$

where $t$ is the number of tied observation sets, $t_{i}$ is the number of tied observations in the TRUE ranking, and $u_{i}$ is the number of tied observations in the ranking derived from the method.

We ran 20,000 trials for each of the 30 design scenarios, and replications were parallelized to save computational resources, mainly time. 
Table 1 and Fig. 4 show the average hit ratio for each of the 30 design elements, i.e., the average values of 20,000 trials, considering a risk-neutral DM. We have marked the maximum hit ratio for each method across all 30 design scenarios in bold. The labels along the abscissa of the chart in Fig. 4 consist of two values corresponding to the number of alternatives and attributes, respectively. There are four columns for each label, representing the hit ratio or rank-order correlation levels for the considered methods.

Fig. 4 shows that the hit ratio decreases as the number of alternatives that there are for any given number of attributes grows, which is obvious. Additionally, the number of attributes also affects the hit ratio; it is greater the more attributes there are for any given number of alternatives.

$D M E 1$ and DME2 methods clearly outperform the results provided by AP1 in all scenarios. DME1 and DME2 are much better than $A P 1$ when there are a lot of alternatives. These results are consistent with the findings reported in $\langle 16\rangle$, in which imprecision is represented by value intervals in alternative performances, component values and weights, and $\langle 13\rangle$, in which ordinal information is considered for weights. The difference in the mean hit ratios between $D M E 2$ and $A P 1$ is $1.236 \%$ for three and $6.262 \%$ for twenty alternatives.

We also find that the proposed method, DIM, outputs better results than the DME1 and DME2 methods in all scenarios. DIM performs much better than $D M E 1$ and DME2 at larger numbers of alternatives. The mean hit ratio is 81.38 for $D I M$ and 77.24 and 78.39 for $D M E 1$ and DME2, respectively.

$D I M$ outputs very similar results to the $S D$ method, and the difference between the average hit ratio is only 0.02. DIM outperforms the $S D$ method in 10 scenarios, but the difference is lower than 0.54 in all cases. However, there are also five cases in which $S D$ outperforms the DIM method, but now the difference is less than 0.02 .

Furthermore, according to the paired-samples t-test (which computes the difference between the mean values of the two methods and tests whether the average differs from zero), there is no significant difference between the hit ratio means of the $D I M$ and $S D$ methods depending of the value of the significance level (significance level, two-tailed: 0.02546).

Table 2 and Fig. 4 show the rank-order correlations for each of the 30 design elements for a risk-neutral DM. Fig. 4 shows that the rank-order correlations increases proportionally to the number of attributes. Besides, the rank-order correlations for the DME2, DIM and SD methods also increases proportionally to the number of attributes.

$D I M$ again outperforms DME1 and DME2 in all the scenarios, which also outperfoms $A P 1$. The results output by the $S D$ and DIM methods are again very similar, the difference between the average rank-order correlations being only 0.05. There are scenarios in which the DIM method is better than $S D$ method, mainly when there are not many attributes, whereas $S D$ slightly outperforms the DIM method for 10 or more alternatives. However, the difference is always lower than 0.02 . 


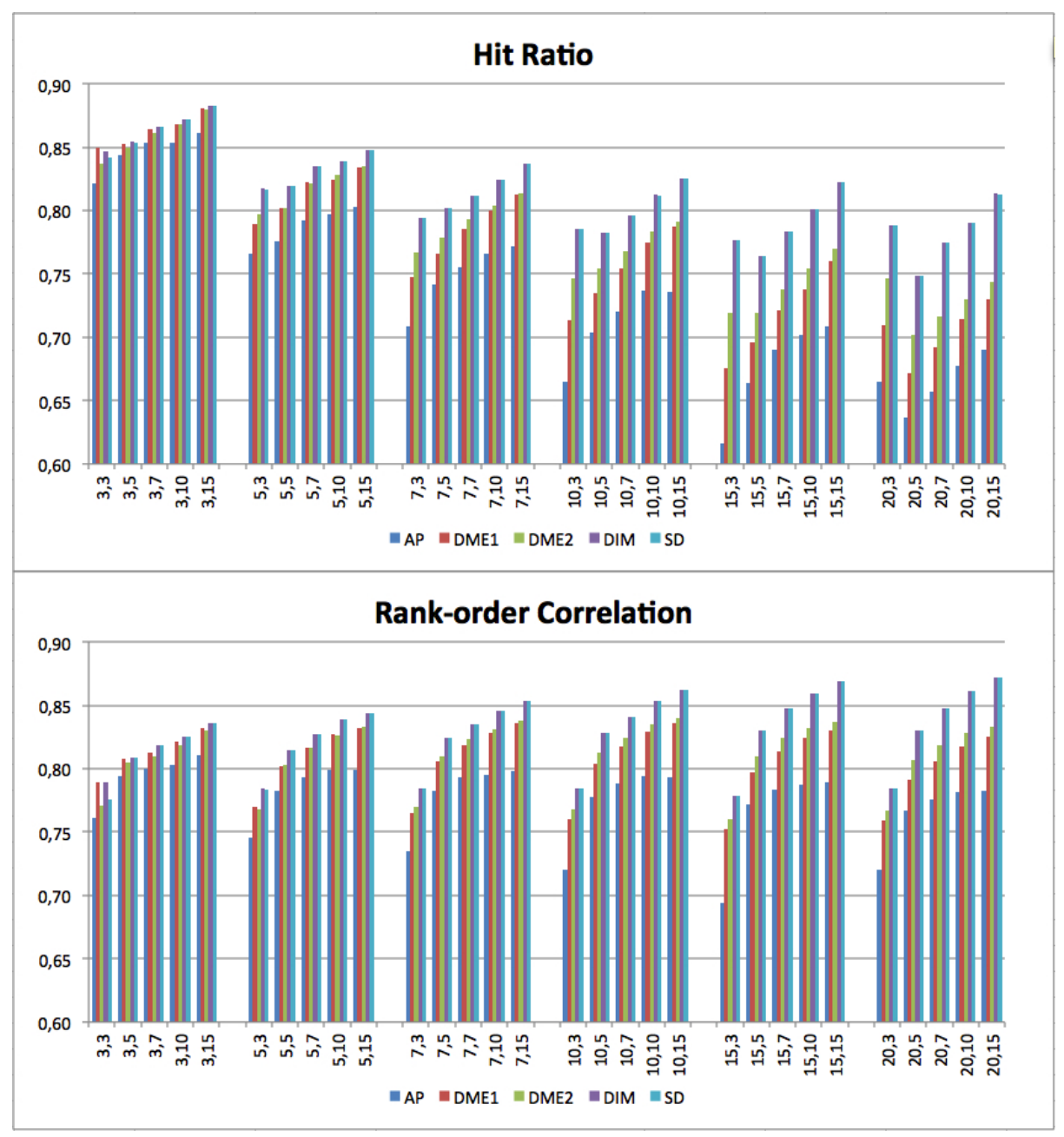

Figure 4: Hit ratio and rank-order correlation levels. 
According to the paired-samples $t$-test, there is no significant difference between the hit ratio means (significance level, two-tailed: 0.064838).

The results for risk-prone and risk-averse DMs are similar. Table 3 shows the average hit ratios and rank-order correlations for both situations. Maximum values are marked in bold and correspond to the DIM method in all cases. The DIM method again outputs better results than the DME1 and DME2 methods, which are better than $A P 1$. The $S D$ and $D I M$ methods are again very similar, and the difference between the hit ratio and rank-order correlation means of the $D I M$ and $S D$ methods are not significant (significance levels, two-tailed: 0.027587 and 0.057604 , respectively, for a risk-prone DM; and 0.023705 and 0.077825 , for a risk-adverse DM). 
Table 1. Hit ratios. Risk-neutral DM

\begin{tabular}{|c|c|c|c|c|c|c|}
\hline \multirow[t]{2}{*}{ Alternatives } & \multirow[t]{2}{*}{ Criteria } & \multicolumn{4}{|c|}{ Methods } & \multirow[b]{2}{*}{$S D$} \\
\hline & & $A P 1$ & $D M E 1$ & $D M E 2$ & $D I M$ & \\
\hline \multirow[t]{5}{*}{3} & 3 & 82.13 & 84.91 & 83.73 & 84.68 & 84.14 \\
\hline & 5 & 84.42 & 85.21 & 84.92 & 85.41 & 85.36 \\
\hline & 7 & 85.32 & 86.39 & 86.12 & 86.62 & 86.64 \\
\hline & 10 & 85.37 & 86.79 & 86.82 & 87.16 & 87.15 \\
\hline & 15 & 86.12 & 88.09 & 87.95 & 88.30 & 88.29 \\
\hline \multirow[t]{5}{*}{5} & 3 & 76.56 & 78.91 & 79.72 & 81.74 & 81.68 \\
\hline & 5 & 77.57 & 80.24 & 80.22 & 81.92 & 81.92 \\
\hline & 7 & 79.22 & 82.27 & 82.13 & 83.46 & 83.46 \\
\hline & 10 & 79.72 & 82.43 & 82.77 & 83.90 & 83.90 \\
\hline & 15 & 80.25 & 83.43 & 83.49 & 84.76 & 84.76 \\
\hline \multirow[t]{5}{*}{7} & 3 & 70.83 & 74.79 & 76.65 & 79.42 & 79.41 \\
\hline & 5 & 74.19 & 76.64 & 77.83 & 80.19 & 80.19 \\
\hline & 7 & 75.50 & 78.56 & 79.28 & 81.21 & 81.21 \\
\hline & 10 & 76.59 & 80.02 & 80.41 & 82.40 & 82.40 \\
\hline & 15 & 77.14 & 81.29 & 81.38 & 83.70 & 83.70 \\
\hline \multirow[t]{5}{*}{10} & 3 & 66.44 & 71.35 & 74.65 & 78.58 & 78.57 \\
\hline & 5 & 70.40 & 73.52 & 75.39 & 78.26 & 78.26 \\
\hline & 7 & 72.05 & 75.38 & 76.80 & 79.64 & 79.64 \\
\hline & 10 & 73.72 & 77.43 & 78.37 & 81.22 & 81.20 \\
\hline & 15 & 73.58 & 78.72 & 79.08 & 82.51 & 82.51 \\
\hline \multirow[t]{5}{*}{15} & 3 & 61.62 & 67.52 & 71.95 & 77.68 & 77.67 \\
\hline & 5 & 66.34 & 69.64 & 71.93 & 76.39 & 76.40 \\
\hline & 7 & 68.97 & 72.08 & 73.80 & 78.38 & 78.38 \\
\hline & 10 & 70.20 & 73.80 & 75.46 & 80.12 & 80.12 \\
\hline & 15 & 70.86 & 76.00 & 76.96 & 82.26 & 82.27 \\
\hline \multirow[t]{5}{*}{20} & 3 & 66.44 & 71.00 & 74.70 & 78.85 & 78.84 \\
\hline & 5 & 63.63 & 67.17 & 70.18 & 74.84 & 74.86 \\
\hline & 7 & 65.70 & 69.25 & 71.66 & 77.42 & 77.42 \\
\hline & 10 & 67.78 & 71.46 & 73.01 & 79.06 & 79.07 \\
\hline & 15 & 69.03 & 72.98 & 74.34 & 81.31 & 81.29 \\
\hline Mean & & 73.92 & 77.24 & 78.39 & 81.38 & 81.36 \\
\hline
\end{tabular}


Table 2. Rank-order correlation (Kendall's $\tau$ ). Risk-neutral DM

\begin{tabular}{|c|c|c|c|c|c|c|}
\hline \multirow[t]{2}{*}{ Alternatives } & \multirow[t]{2}{*}{ Criteria } & \multicolumn{4}{|c|}{ Methods } & \multirow[b]{2}{*}{$S D$} \\
\hline & & $A P 1$ & $D M E 1$ & $D M E 2$ & $D I M$ & \\
\hline \multirow[t]{5}{*}{3} & 3 & 76.12 & 78.97 & 77.12 & 78.93 & 77.55 \\
\hline & 5 & 79.42 & 80.74 & 80.45 & 80.91 & 80.89 \\
\hline & 7 & 79.99 & 81.23 & 80.96 & 81.83 & 81.82 \\
\hline & 10 & 80.32 & 82.12 & 81.81 & 82.48 & 82.48 \\
\hline & 15 & 81.06 & 83.16 & 83.01 & 83.63 & 83.63 \\
\hline \multirow[t]{5}{*}{5} & 3 & 74.53 & 76.98 & 76.83 & 78.40 & 78.34 \\
\hline & 5 & 78.24 & 80.22 & 80.33 & 81.44 & 81.43 \\
\hline & 7 & 79.36 & 81.67 & 81.63 & 82.72 & 82.72 \\
\hline & 10 & 79.92 & 82.68 & 82.64 & 83.90 & 83.90 \\
\hline & 15 & 79.91 & 83.23 & 83.28 & 84.41 & 84.41 \\
\hline \multirow[t]{5}{*}{7} & 3 & 73.45 & 76.47 & 76.95 & 78.45 & 78.43 \\
\hline & 5 & 78.24 & 80.62 & 81.01 & 82.41 & 82.42 \\
\hline & 7 & 79.28 & 81.82 & 82.34 & 83.46 & 83.46 \\
\hline & 10 & 79.50 & 82.84 & 83.07 & 84.52 & 84.52 \\
\hline & 15 & 79.75 & 83.63 & 83.80 & 85.31 & 85.31 \\
\hline \multirow[t]{5}{*}{10} & 3 & 72.05 & 75.98 & 76.81 & 78.41 & 78.42 \\
\hline & 5 & 77.79 & 80.40 & 81.23 & 82.77 & 82.77 \\
\hline & 7 & 78.85 & 81.75 & 82.44 & 84.07 & 84.07 \\
\hline & 10 & 79.40 & 82.92 & 83.47 & 85.34 & 85.34 \\
\hline & 15 & 79.35 & 83.60 & 84.01 & 86.23 & 86.23 \\
\hline \multirow[t]{5}{*}{15} & 3 & 69.43 & 75.25 & 76.03 & 77.84 & 77.87 \\
\hline & 5 & 77.15 & 79.71 & 80.98 & 83.00 & 83.01 \\
\hline & 7 & 78.29 & 81.34 & 82.40 & 84.73 & 84.74 \\
\hline & 10 & 78.76 & 82.38 & 83.23 & 85.89 & 85.89 \\
\hline & 15 & 78.91 & 83.03 & 83.67 & 86.91 & 86.91 \\
\hline \multirow[t]{5}{*}{20} & 3 & 72.00 & 75.93 & 76.72 & 78.40 & 78.41 \\
\hline & 5 & 76.65 & 79.16 & 80.64 & 83.03 & 83.04 \\
\hline & 7 & 77.57 & 80.57 & 81.88 & 84.76 & 84.78 \\
\hline & 10 & 78.14 & 81.79 & 82.78 & 86.10 & 86.11 \\
\hline & 15 & 78.29 & 82.53 & 83.29 & 87.20 & 87.20 \\
\hline Mean & & 77.72 & 80.76 & 81.16 & 82.92 & 82.87 \\
\hline
\end{tabular}

Table 3. Results for a risk-prone and a risk-adverse DM

\begin{tabular}{ccccccc}
\hline & Measure & $A P 1$ & $D M E 1$ & $D M E 2$ & $D I M$ & $S D$ \\
\hline Risk-prone & Hit ratio & 73.78 & 77.10 & 78.30 & 81.27 & 81.25 \\
& Kendall's $\tau$ & 77.69 & 80.743 & 81.16 & 82.89 & 82.85 \\
\multirow{2}{*}{ Risk-adverse } & Hit ratio & 73.90 & 77.24 & 78.38 & 81.32 & 81.30 \\
& Kendall's $\tau$ & 77.70 & 80.74 & 81.18 & 82.92 & 82.86 \\
\hline
\end{tabular}




\section{Conclusions}

We have proposed a new dominance measuring method to deal with ordinal information about the decision-maker's preferences, in both weights and component values. The decision maker provides a ranking of attribute importance. Besides, the method takes into account a ranking of the alternatives in each attribute and also a ranking of the difference of values between consecutive alternatives.

The proposed method uses the centroid of the polytope delimited by ordinal information and builds triangular fuzzy numbers, whose distances to the crisp value 0 are the basis for the definition of a dominance intensity measure.

The results of Monte Carlo simulation techniques applied demonstrate that the proposed method is clearly better at selecting the best alternative and ranking alternatives than other dominance measuring methods proposed in the literature. Its performance is very similar to the method proposed by Sarabando and Dias, which was developed to deal with decision-making problems with ordinal information about the decision-maker's preferences too. The paired-samples $t$ test shows that there is no significant difference between the two for a neutral, risk-prone and risk-averse decision-maker.

Sarabando and Dias's method is less computationally demanding, but its application is restricted to the discussed imprecise decision-making situation. On the other hand, the method proposed in this paper can also be used if we have the ranking of alternatives for each attribute under consideration, but the information about the differences between the values of consecutive alternatives is not available. The algorithm proposed to derive endpoints in the centroid computation still works. This situation is less stressful on DMs and makes the method suitable for much more real decision-making problems.

As a future research line we propose the use of simulation techniques to approximate the centroid and conduct the respective analysis of its performance when different types of partial ordinal information are available. Moreover, we

also intend to use other types of fuzzy sets and different notions of associated distances to derive the final ranking of alternatives.

\section{References}

[1] B.S. Ahn, Extending Malakooti's model for ranking multicriteria alternatives with preference strength and partial information, IEEE Trans. Syst., Man. Cyb.: Part A 33 (2003) 281-287.

[2] B.S. Ahn, K.S. Park, Comparing methods for multiattribute decision making with ordinal weights, Comput. Oper. Res. 35 (2008) 1660-1670.

[3] F. Barron, B. Barret, Decision quality using ranked attribute weights. Man. Sci. 42 (1996) 1515-1523.

[4] D. Dubois, H. Prade, Fuzzy sets and systems: theory and applications, Academic Press, New York, 1980. 
[5] J. Jia, G.W. Fischer, J.S. Dyer, Attribute weighting method and decision quality in the presence of response error: A simulation study. J. Behav. Decis. Making 11(1998) 85-105.

[6] A. Jiménez, A. Mateos, S. Ríos Insua, Monte Carlo simulation techniques in a decision support system for group decision-making. Group Decis. Negotiation 14 (2005) 109-130.

[7] A. Jiménez, A. Mateos, P. Sabio, Dominance intensity measure within fuzzy weight oriented MAUT: an application. Omega 41(2013) 397-405.

[8] R. Lahdelma, J. Hokkanen, P. Salminen, SMAA-Stochastic multiobjective acceptability analysis. Eur. J. Oper. Res. 106 (1998) 137-143.

[9] R. Lahdelma, K. Miettinen, P. Salminen, Ordinal criteria in stochastic multi-criteria acceptability analysis (SMAA). Eur. J. Oper. Res. 147 (2003) $117-127$.

[10] R. Lahdelma, P. Salminen, SMAA-2: Stochastic multicriteria acceptability analysis for group decision making. Oper. Res. 49 (2001) 444-454.

[11] B. Malakooti, Ranking and screening multiple criteria alternatives with partial information and use of ordinal and cardinal strength of preferences. IEEE Trans. Syst., Man. Cyb.: Part A 30 (2000) 787-801.

[12] A.M. Mármol, J. Puerto, F.R. Fernández, The use of partial information on weights in multicriteria decision problems. J. Multi-Crit. Decis. Anal. 7 (1998) 322-329.

[13] A. Mateos, A. Jiménez, E.A. Aguayo, P. Sabio, Dominance intensity measuring methods in MCDM with ordinal relations regarding weights. Knowl. Based Syst. (2013) DOI:10.1016/j.kynosys.2013.12.002.

[14] A. Mateos, A. Jiménez, E.A. Aguayo, P. Sabio, Preference intensity in MCDM when and additive utility function represents DM preferences. In: C. Kahraman, E.E. Kerre and P.T. Bozbura (eds.), Uncertainty Modelling in Knowledge Engineering and Decision Making, World Scientific Proceeding Series on Computing Engineering and Information Systems, 2012, pp. 106-111, World Scientific, New Jersey.

[15] A. Mateos, A. Jiménez, J.F., Blanco, Ranking methods based on dominance measures accounting for imprecision. In: F. Rossi and A. Tsoukis (eds.), Lecture Notes on Artificial Intelligence 5783, 2009, pp. 328-339, Springer, Berlin.

[16] A. Mateos, A. Jiménez, J.F. Blanco, Measuring method performance under incomplete information about weights. J. Multi-Crit. Decis. Anal. 19 (2012) 129-142. 
[17] A. Mateos, A. Jiménez, S. Ríos Insua, S., Monte Carlo simulation techniques for group decision-making with incomplete information. Eur. J. Oper. Res. 174 (2006) 1842-1864.

[18] P. Nijkamp, P. Rietveld, H. Voogd, Multicriteria evaluation physical planning, North Holland, Amsterdam, 1990.

[19] J. Puerto, A.M. Mármol, L. Monroy, F.R. Fernández, F.R., Decision criteria with partial information. IEEE Trans. Oper. Res. 7(2000) 51-65.

[20] H. Raiffa, The Art and Science of Negotiation, Harvard University Press, Cambridge, 1982.

[21] D. Ríos Insua, Sensitivity Analysis in Multi-objective Decision Making, Springer, New York, 1990.

[22] D. Ríos, S. French, A framework for sensitivity analysis in discrete multiobjective decision-making. Eur. J. Oper. Res. 54 (1991) 176-190.

[23] B. Roy, V. Mousseau, A theoretical framework for analysing the notion of relative importance of criteria. J. Multi-Crit. Decis. Anal. 5 (1996) 1451159.

[24] A. Sage, C.C. White, Ariadne: a knowledge-based interactive system for planning and decision support. IEEE Trans. Syst., Man. Cyb.: Part A 14 (1984) 35-47.

[25] A. Salo, R.P. Hamalainen, Preference ratio in multiattribute evaluation (PRIME) - elicitation and decision procedures under incomplete information. IEEE Trans. Syst., Man. Cyb.: Part A 31(2001) 533-545.

[26] P. Sarabando, L.C. Dias, Multi-attribute choice with ordinal information: a comparison of different decision rules. IEEE Trans. Syst., Man. Cyb.: Part A 39 (2009) 545-554.

[27] P. Sarabando, L.C. Dias, Simple procedures of choice in multicriteria problems without precise information about the alternatives values, Comput. Oper. Res. 37 (2010) 2239-2247.

[28] T. Solymosi, J. Dombi, A method for determining the weights of criteria: the centralized weights. Eur. J. Oper. Res. 26 (1986) 3541.

[29] T.J. Stewart, Robustness of additive value function method in MCDM. J. Multi-Crit. Decis. Anal. 5 (1996) 301-309.

[30] W.G. Stillwell, D.A. Seaver, W.A. Edwards, Comparison of weight approximation techniques in multiattribute utility decision making. Org. Behav. Human Decis. Proc. 28 (1981) 62-77.

[31] L. Tran, L. Duckstein, Comparison of fuzzy numbers using a fuzzy distance measure. Fuzzy Sets Syst. 130 (2002) 331-341. 
[32] R. Vertschera, M. Filzmoser, R. Mittechofer, An analytical approach to offer generation in concession-based negotiation processes. Group Dec. and Neg. 23, 2 (2014) 71-99.

[33] M. Weber Decision making with incomplete information. Eur. J. Oper. Res. 28 (1987) 44-57.

[34] R.L. Winkler, W.L. Hays, Statistics: probability, inference and decision, Holt, Rinehart \& Winston, New York, 1985.

[35] Z. Xu, A method for multiple attribute decision making with incomplete weight information in linguistic setting. Knowl-Based Syst. 20 (2007) 719725 .

[36] Y. Xu, Q. Da, A method for multiple attribute decision making with incomplete weight information under uncertain linguistic environment. KnowlBased Syst. 21, 2008, 837-841.

[37] Y.J. Xu, J.N.D. Gupta, H.M. Wang, The ordinal consistency of an incomplete reciprocal preference relation. Fuzzy Sets Syst. 246 (2014) 62-77.

[38] Y.J. Xu, F. Ma, F.F. Tao, H.M. Wang, Some methods to deal with unacceptable incomplete 2-types fuzzy linguistic preference relations in group decision making, Knowl-Based Syst. 56 (2014) 179-190.

[39] Y.J. Xu, R. Patnayakuni, H.M. Wang, The ordinal consistency of a fuzzy preference relation, Inform. Sc. 224, 1 (2013) 152-164.

[40] Y.J. $\mathrm{Xu}, \quad$ H.M. Wang, D.J. Yu, Weak transitivity of interval-valued relations, Knowl-Based Syst. (2014) http://dx.doi.org/10.1016/j.knosys2014.03.003

[41] Z. Yue, An extended TOPSIS for determining weights of decision makers with interval numbers. Knowl-Based Syst. 24 (2011) 146-153. 